Portland State University

PDXScholar

Mechanical and Materials Engineering Faculty

Publications and Presentations

$11-2001$

\title{
Capillary Flow in Interior Corners: The Infinite Column
}

Mark M. Weislogel

Portland State University, weisloge@pdx.edu

Follow this and additional works at: https://pdxscholar.library.pdx.edu/mengin_fac

Part of the Fluid Dynamics Commons, and the Materials Science and Engineering Commons Let us know how access to this document benefits you.

Citation Details

Weislogel, M. M. (2001). Capillary flow in interior corners: The infinite column. Physics Of Fluids, 13(11), 3101.

This Article is brought to you for free and open access. It has been accepted for inclusion in Mechanical and Materials Engineering Faculty Publications and Presentations by an authorized administrator of PDXScholar. Please contact us if we can make this document more accessible: pdxscholar@pdx.edu. 


\title{
ARTICLES
}

\section{Capillary flow in interior corners: The infinite column}

\author{
M. M. Weislogel ${ }^{\text {a) }}$ \\ TDA Research, Inc., 12345 W. 52nd Avenue, Wheat Ridge, Colorado 80033
}

(Received 28 August 2000; accepted 10 July 2001)

Capillary flow of a sinusoidally perturbed liquid column in an interior corner of infinite extent is solved using lubrication theory. Due primarily to the length scales selected to nondimensionalize the momentum equation, an analytic time scale governing the settling of the perturbation is determined. The time scale, which is shown to be independent of a steady base state flow, proves useful in rapidly predicting transients for surface settling in certain liquid-bearing tanks of spacecraft employing interior corners for fluids management purposes. The asymptotic analysis is extended to address flows along interior corners whose faces are slightly nonplanar. The generalized formulation is presented for the case of a perfectly wetting fluid in a second-order polynomial corner. A leading-order analytic solution for small corner angles is provided. It is shown that a "convex corner" decreases the response time of the liquid and increases the capillary flow rate along the corner by increasing both the driving force and cross-sectional area of the flow. Gravity acting normal to the corner axis along the bisector of the corner angle is also considered and is found to accelerate, decelerate, or destabilize such flows depending on its sign and magnitude. (C) 2001 American Institute of Physics. [DOI: 10.1063/1.1408918]

\section{BACKGROUND}

A wetting phenomenon of fundamental importance first described with mathematical rigor by Concus and Finn ${ }^{1}$ occurs in containers with interior corners for corner half-angles $\alpha$ and contact angles $\theta$ that satisfy $\theta<\pi / 2-\alpha$. The ConcusFinn condition is a geometric wetting condition that corresponds to an underpressure in the liquid resulting in capillary-driven flow into and along the interior corner. A column of liquid stably occupies an infinite interior corner when the Concus-Finn condition is satisfied but is unstable when the condition is not satisfied. As shown by Langbein, ${ }^{2}$ the latter situation produces an overpressure in the liquid similar to that of a cylindrical jet, which breaks up into finite "drops" whose shapes and orientations depend on $\alpha, \theta$, and liquid volume. When the Concus-Finn condition is satisfied, the interior corners of containers may be used to move, preferentially locate, or otherwise control capillary liquids.

In the absence of gravity, spontaneous capillary flows occur over large length scales on the order of greater than 1 $\mathrm{m}$. As a result, spacecraft fluids systems can exploit capillary flows in interior corners for passive positioning and/or flow control of large liquid masses such as cryogens, storable fuels, and water. Because numerical methods are difficult to apply in capillary systems, analytic tools to predict such fluid behavior are desired to improve design efficiency with regard to both time and cost.

a) Telephone: 303-940-2320; fax: 303-422-7763; electronic mail: mmw@tda.com
A general formulation for these capillary flows based on lubrication theory was outlined by Weislogel and Lichter ${ }^{3}$ (W\&L) providing the framework for analytical solutions to a variety of application-specific problems. Because slender liquid columns in interior corners often arise in low-g fluids management systems, an effort is made here to apply the general formulation to this important problem. A brief review is provided below by way of a description of the infinite corner flow.

\section{THE INFINITE COLUMN}

In Fig. 1 is sketched a portion of an interior corner formed by planar walls of infinite extent. The corner is partially filled with a wetting liquid of viscosity $\mu$ and surface tension $\sigma$ and satisfies the Concus-Finn condition for this geometry: namely, $\theta<\pi / 2-\alpha$, where $\theta$ is the wetting angle of the liquid on the solid faces of the corner and $\alpha$ is the corner half-angle. Here $H$ is the characteristic height of the liquid in the corner, and the liquid surface $S_{0}^{\prime}\left(x^{\prime}, z^{\prime}, t^{\prime}\right)$ is given an axial perturbation characterized by wavelength $2 L$. The slenderness ratio $\epsilon=H / L$ appears as $\epsilon^{2}$ in the nondimensional equations and serves as the small parameter, allowing asymptotic analysis of the problem.

As reviewed by W\&L, ${ }^{3}$ the assumption $\epsilon^{2} \ll 1$ simplifies the normal stress boundary condition on the fluid interface to the Young-Laplace equation, which may be solved to express the entire fluid interface shape as a construct of circular arcs in the dimensional $x^{\prime}-y^{\prime}$ plane. The local radius of curvature of the interface $R^{\prime}=R^{\prime}\left(z^{\prime}, t^{\prime}\right)$, which is inversely proportional to the pressure $P_{0}^{\prime}=\sigma / R^{\prime}$ in the liquid, may be 


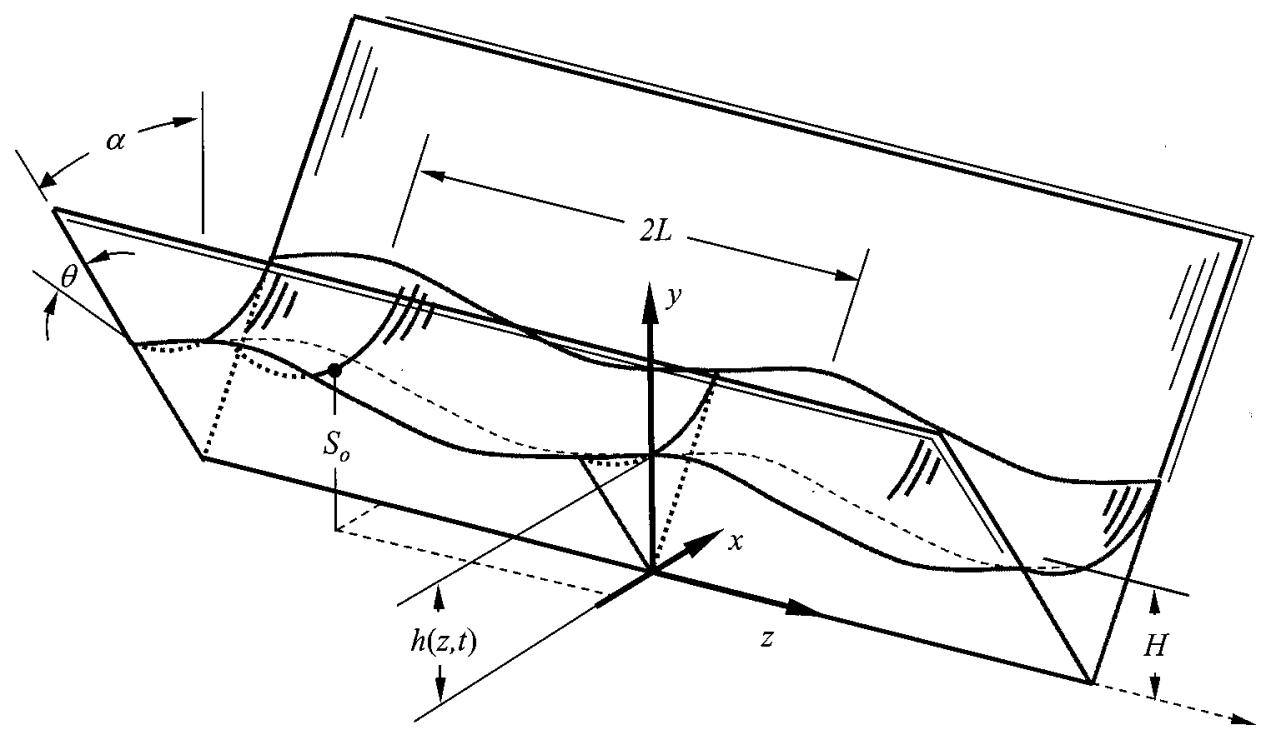

FIG. 1. Perturbed liquid column in an infinite interior corner. The notation identifies dimensional quantities (primes dropped).

expressed in terms of local surface height $h^{\prime}\left(z^{\prime}, t^{\prime}\right)$. The "cross-flow problem" for Fig. 1 is sketched in Fig. 2, where $R^{\prime}$ is constant in the $x^{\prime}-y^{\prime}$ plane and the liquid surface satisfies the static contact angle condition at the wall; Fig. 2(a). Here $R^{\prime}$ is nondimensionalized by $H f$, where

$$
f=\sin \alpha /(\cos \theta-\sin \alpha) .
$$

The radius of curvature in the dimensionless $x$ - $y$ plane is not constant; Fig. 2(b).

For $\epsilon^{2} \ll 1$, the flow is predominantly parallel and the nondimensionalization of the governing momentum and mass equations using the quantities listed in Table I leads to

$$
\frac{\partial P_{0}}{\partial z}=\frac{\partial^{2} w_{0}}{\partial y^{2}} \sin ^{2} \alpha+\frac{\partial^{2} w_{0}}{\partial x^{2}} \cos ^{2} \alpha,
$$

for the leading-order $z$-component velocity distribution $w_{0}$ in the dimensionless $x-y$ cross-flow plane. Equation (1) is subject to no slip along the walls, no shear stress on the free surface, and the symmetry condition along $x=0$. The streamwise mass balance is

$$
\frac{\partial A}{\partial t}=-\frac{\partial \dot{Q}}{\partial z},
$$

where $A$ is the dimensionless cross-flow area and $\dot{Q}$ $=A\left\langle w_{0}\right\rangle$ is the volumetric $z$-component volumetric flow rate, where $\left\langle w_{0}\right\rangle$ is the dimensionless average velocity through the section $A$. The streamwise mass balance may be conveniently written in terms of meniscus centerline height $h(z, t)$, given generally by

$$
\frac{\partial F_{a} h^{2}}{\partial t}=\frac{\partial}{\partial z}\left(F_{a} h^{2} F_{\nu} \frac{\partial h}{\partial z}\right),
$$

where $A \equiv F_{a} h^{2}$ and $\left\langle w_{0}\right\rangle \equiv-F_{\nu} \partial h / \partial z$, the mean $z$-component velocity through the cross-flow section. Here $F_{a}$ is a geometric weighting factor for the cross-flow area and $F_{\nu}$ is the geometric flow resistance. Both $F_{a}$ and $F_{\nu}$ depend on $\alpha$ and $\theta$. For nonplanar walls $F_{a}$ and $F_{\nu}$ also depend on $h$. Solutions of Eq. (3) for $h(z, t)$ may be used to construct the transient three-dimensional (3-D) interface shape, $S(x, z, t)$.

Numerous researchers have followed the arguments leading to Eq. (3) for capillary flows in corners, and a selection of recent examples includes the work of Gauglitz et al., ${ }^{4}$ Dong and Chatzis, ${ }^{5}$ and Langbein and Weislogel. ${ }^{6}$ The subtle novelty of the present development is the nondimensionalization of the problem, which explicitly accounts for much of the variation of $F_{\nu}$ on $\alpha$ and $\theta$, significantly reducing the dependence of analytical results on numerical solutions to Eq. (1), or the use of friction factors or hydraulic diameters. For example, the $z^{\prime}$-component velocity scale $W$ is deter-
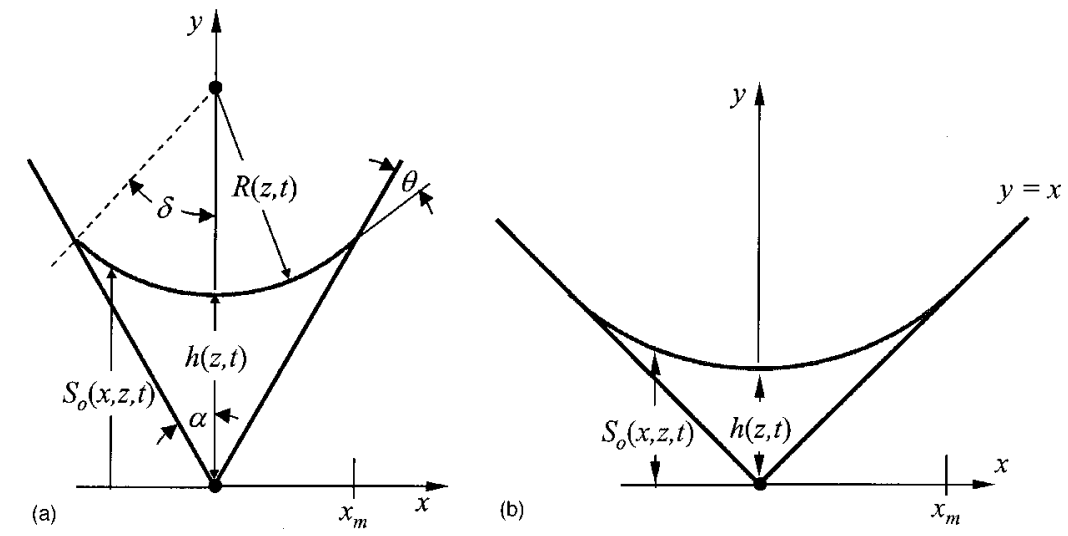

FIG. 2. Cross-flow notation: (a) dimensional (primes dropped); (b) nondimensional. 
TABLE I. Nondimensionalized terms, parameters, and definitions used in analyses. Primes denote dimensional quantities.

\begin{tabular}{lll}
\hline \hline Lengths & \multicolumn{1}{c}{ Other } & \multicolumn{1}{c}{ Geometric parameters, definitions } \\
\hline$x=x^{\prime} / H \tan \alpha$ & $w_{0}=w_{0}^{\prime} / W$ & $F_{a}$, geometric cross flow are function \\
$y=y^{\prime} / H$ & $W=\epsilon \sigma \sin ^{2} \alpha / \mu f$ & $F_{a}=F_{A} / \tan \alpha$, planar corner \\
$z=z^{\prime} / L$ & $t=W t^{\prime} / L$ & $F_{a}=1 \mp 2 \beta h / 3$, nonplanar corner, $\alpha^{2} \ll 1$, Eq. (30) \\
$h=h^{\prime} / H$ & $P_{0}=f H P_{0}^{\prime} / \sigma$ & $F_{A}=f^{2}(\cos \theta \sin \delta / \sin \alpha-\delta)$ \\
$R=R^{\prime} / f H$ & $A=A^{\prime} / H^{2} \tan \alpha$ & $F_{\nu}$, generalized geometric flow resistance parameter \\
$S_{0}=S_{0}^{\prime} / H$ & $\dot{Q}=\dot{Q}^{\prime} / W H^{2} \tan \alpha$ & $F_{i}$, geometric flow resistance for planar corner \\
$f_{w}=f_{w}^{\prime} / H$ & & $F_{\nu}=F_{i}$, planar corner, $\frac{1}{8}<F_{i}<\frac{1}{6}$, W\&L (Ref. 3) \\
& & $F_{i}=F_{I}=\frac{1}{6}+\frac{\alpha}{3}\left(1-\frac{5 \pi}{16}\right)$ planar corner, $\alpha^{2} \ll 1$, W\&L (Ref. 3) \\
& & $F_{\nu}=F_{I} \mp 13 \beta / 45$, nonplanar corner, $\alpha^{2} \ll 1$, Eq. (29) \\
\hline \hline
\end{tabular}

mined by a balance of surface tension and viscous forces in the following manner: The dimensional equation for the cross-flow problem is $P_{0 z}=\mu\left(w_{0 x x}+w_{0 y y}\right)$. Substituting the Table I length and pressure scales produces the balance

$$
\frac{\sigma}{H f L} \sim \mu W\left(\frac{1}{H^{2} \tan ^{2} \alpha}+\frac{1}{H^{2}}\right)=\frac{\mu W}{H^{2} \sin ^{2} \alpha} .
$$

Solving for $W$ yields the velocity scale $W=\epsilon \sigma \sin ^{2} \alpha / \mu f$ listed in Table I. As a consequence of this scaling, surface tension forces depend on both $\alpha$ and $\theta$ through $1 / f$, while viscous forces depend primarily on $\alpha$ through $1 / \sin ^{2} \alpha$. The degree to which this scaling models the flow is discussed further below.

\section{INFINITE COLUMN: PLANAR WALLS}

For planar walls as sketched in Figs. 1 and $2, F_{a}$ $=F_{A}(\alpha, \theta)$ and $F_{\nu}=F_{i}(\alpha, \theta)$. Thus, for this case, $F_{a}$ divides out of Eq. (3). The exact value of $F_{\nu}$ must be determined numerically, as accomplished by Ransohoff and $\operatorname{Radke}^{7}$ and Ayyaswamy et $a l .{ }^{8}$ However, as shown by W\&L, ${ }^{3}$ analytical solutions to Eq. (1) for small and large corner angles reveal that $1 / 8 \leqslant F_{i} \leqslant 1 / 16$ for all values of $\alpha$ and $\theta$ and may therefore be approximated as a constant. By rescaling $\tau=F_{\nu} t / 2$, Eq. (3) becomes

$$
\frac{\partial h}{\partial \tau}=2\left(\frac{\partial h}{\partial z}\right)^{2}+h \frac{\partial^{2} h}{\partial z^{2}} .
$$

For planar corner walls, any solution to Eq. (4) for $h$ leads immediately to resolution of the entire surface through

$$
S_{0}=h(1+f)-\left(f^{2} h^{2}-x^{2} \tan ^{2} \alpha\right)^{1 / 2} .
$$

The above results are accurate to $O(\epsilon)$ provided the column is slender, $\epsilon^{2} \ll 1$. The static contact angle condition is maintained in the cross-flow problem (see Fig. 2) and is correct to leading order, as discussed by Weislogel. ${ }^{9}$

Introducing the expansion $h=h_{0}+\epsilon h_{1}+O\left(\epsilon^{2}\right)$ and substituting into Eq. (4) yields the $O(1)$ equation,

$$
\frac{\partial h_{0}}{\partial \tau}=2\left(\frac{\partial h_{0}}{\partial z}\right)^{2}+h_{0} \frac{\partial^{2} h_{0}}{\partial z^{2}},
$$

and the $O(\epsilon)$ equation,

$$
\frac{\partial h_{1}}{\partial \tau}=4 \frac{\partial h_{0}}{\partial z} \frac{\partial h_{1}}{\partial z}+h_{0} \frac{\partial^{2} h_{1}}{\partial z^{2}}+h_{1} \frac{\partial^{2} h_{0}}{\partial z^{2}} .
$$

One solution to Eq. (6) is $h_{0}=1$. This "base state" solution describes a quiescent planar interface of dimensional height $H$. The $O(\epsilon)$ perturbation Eq. (7) reduces to

$$
\frac{\partial h_{1}}{\partial \tau}=h_{0} \frac{\partial^{2} h_{1}}{\partial z^{2}} .
$$

Equation (8) yields the solution for $h$,

$$
h=1+\epsilon C_{1} \exp \left[-\pi^{2} \tau\right] \cos \left(\pi z+C_{2}\right)+O\left(\epsilon^{2}\right),
$$

which describes the infinite interior corner flow problem sketched in Fig. 1, where $H$ defines the mean (unperturbed) height of the liquid in the corner and $\epsilon C_{1}$ defines the amplitude of an axial sinusoidal perturbation of dimensional wavelength $2 L$. Several useful characteristics of the flow may be distinguished from this result.

An important result from Eq. (9) is the time constant. When redimensionalized, the decaying exponent is

$$
\pi^{2} \tau=\epsilon \frac{\pi^{2}}{2} \frac{F_{\nu} \sin ^{2} \alpha}{f} \frac{\sigma t^{\prime}}{\mu L},
$$

from which the time constant is $2 \mu f L / \pi^{2} \epsilon F_{\nu} \sigma \sin ^{2} \alpha$. For fixed fluid properties and planar corner walls, $1 / 8 \leqslant F_{\nu}=F_{i}$ $\leqslant \frac{1}{6}$, and the response time of the disturbance is dependent

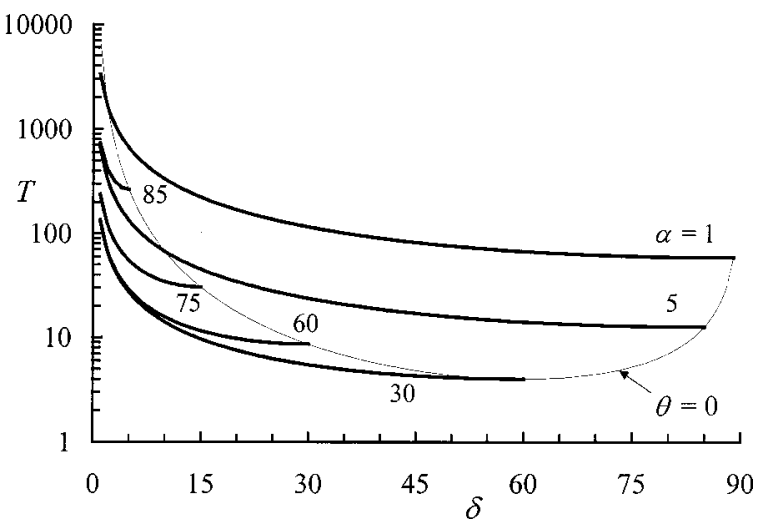

FIG. 3. Geometric time constant $T$ for capillary-driven settling in an infinite corner with planar walls: $\delta \equiv \pi / 2-\alpha-\theta$. The curve for $\theta=0$ is identified by a thin solid line. 


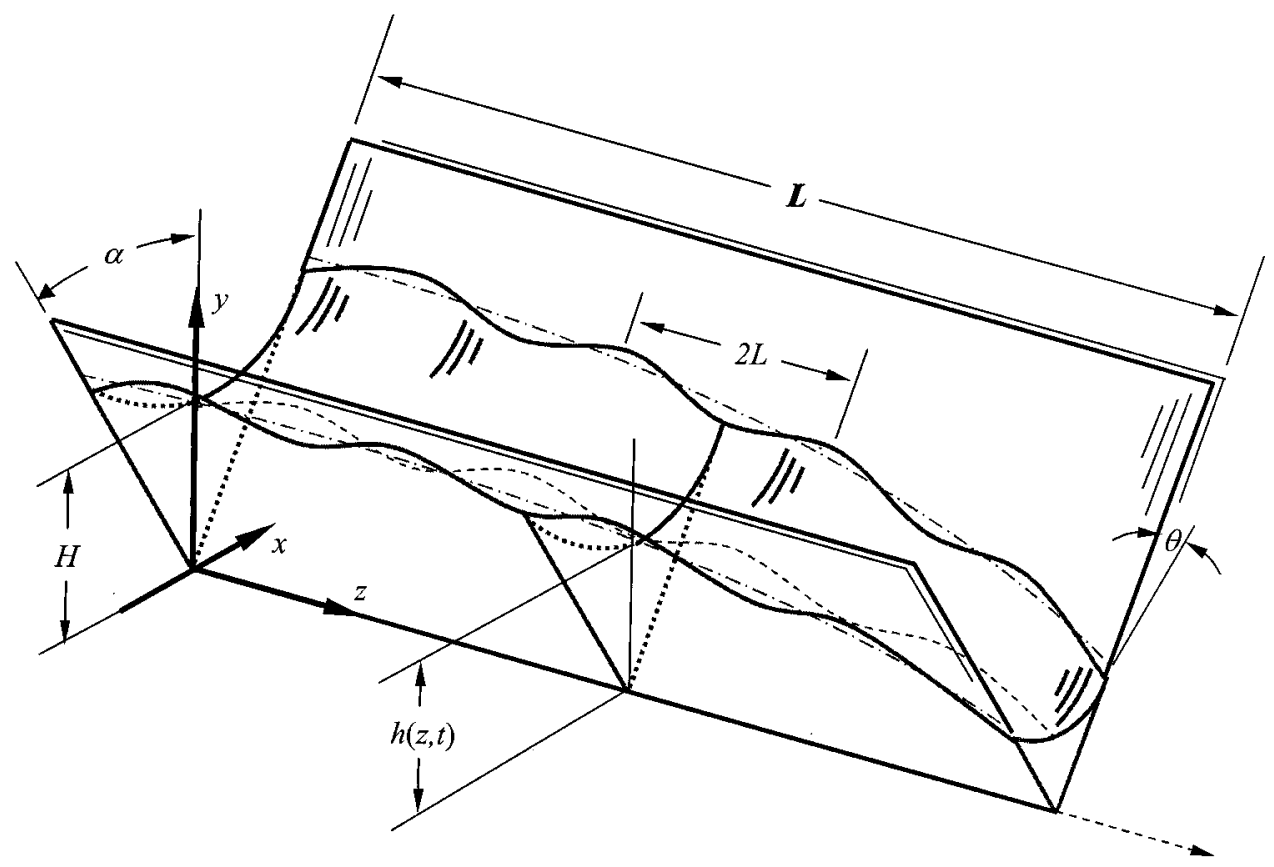

FIG. 4. A sinusoidally perturbed column with a steady flow base state, $h$ $=(1-\Delta z)^{1 / 3}$, where $\Delta=L / \mathbf{L}$. The unperturbed base state is shown using dashed lines on corner walls.

geometrically primarily on the quantity $T=f / \sin ^{2} \alpha$. This relationship is plotted in Fig. 3 for a variety of corner halfangles $\alpha$ and surface curvature angles $\delta[\equiv \pi / 2-\alpha-\theta$; see Fig. 2(a)] satisfying the Concus-Finn condition. As can be seen from the figure, the time constant rapidly increases as $\delta$ approaches 0 . The analytical minimum value for $T$ is achieved for $\alpha=30^{\circ}, \delta=\pi / 2-\alpha$ (i.e., $\theta=0$ ). Even considering the slight variation of $F_{i}$ on $\alpha$ and $\theta$ for the planar corner case, $1 / 8 \leqslant F_{i} \leqslant \frac{1}{6}$, the time constant is minimized for $\alpha=30^{\circ}$, $\delta=\pi / 2-\alpha$. Thus, $T$ serves as the ratio of geometric quantities that characterize capillary $(\sim 1 / f)$ and viscous $\left(\sim 1 / F_{i} \sin ^{2} \alpha\right.$ ) forces. Changes in fluid behavior due to the contact angle are effected largely through the former. The capillary number $\mathrm{Ca}\left(\equiv V^{\prime} \mu / \sigma\right)$ measures the strength of normal viscous to surface tension forces. When based on the dimensional contact line velocity $V^{\prime}=V_{\mathrm{cl}}^{\prime}$, the velocity at the contact line normal to contact line and tangent to the wall, $\mathrm{Ca}$ is often treated as a measure of dynamic contact angle effects as reviewed by Kistler, ${ }^{10}$ which have been assumed negligible. The dimensional leading-order contact line velocity computed from Eq. (9) is $V_{\mathrm{cl}}^{\prime} \approx \partial h^{\prime} / \partial t^{\prime}$. Evaluating $V_{\mathrm{cl}}^{\prime}$ and computing $\mathrm{Ca}$ reveals a maximum value at $t^{\prime}=0$ of $\mathrm{Ca}$ $\sim \epsilon^{3} f /\left(F_{\nu} \sin ^{2} \alpha\right)$, which decreases exponentially with time. This value of $\mathrm{Ca}$ is indeed small and supports the use of the static contact angle condition applied at the contact line for this problem.

\section{A. Steady flow base state}

It is also insightful to note for this formulation that the time constant does not necessarily change for other base states. For example, a steady-state solution to Eq. (6) is $h_{0}$ $=(1-\Delta z)^{1 / 3}$, where $\Delta=L / \mathbf{L}$. This solution introduces the finite length of the liquid column $(\mathbf{L})$ as a second length scale in addition to the disturbance wavelength $(2 L)$ used ex- pressly for the infinite column. For this solution $H$ is the characteristic height of the liquid column. This flow scenario is depicted in Fig. 4 with the base state interface profile identified with a dashed line along the contact line. Using this unperturbed state for $h_{0}$, it is possible for $\Delta^{2} \ll 1$ to show that

$$
\begin{aligned}
h= & (1-\Delta z)^{1 / 3}+\epsilon C_{1} \exp \left[-\pi^{2} \tau\right]\left[\cos \left(\pi z+C_{2}\right)\right. \\
& +\Delta f c n(z)]+O\left(\epsilon^{2}, \Delta^{2}\right),
\end{aligned}
$$

provided $\epsilon \ll \Delta$. Thus the local time dependence of the problem is unchanged by the steady base flow and may be computed using Eq. (10). The impact of base state flow on transients are not expected until $O\left(\epsilon^{2}\right)$, the order of the viscous normal stress condition for $w_{0}$ on $S$.

\section{B. Effect of gravity, $g_{y}$}

The inclusion of gravity acting along the $y$ direction $\left(g_{y}\right.$ taken negative in the negative $y$ direction) for the infinite column problem modifies the mean $z$-component velocity to $\left\langle w_{0}\right\rangle=-F_{\nu}\left(1+h^{2} \mathrm{Bo}_{H}\right) h_{z}$, which when substituted into Eq. (2) leads to

$$
\begin{aligned}
h= & 1+\epsilon C_{1} \exp \left[-\pi^{2}\left(1-\mathrm{Bo}_{H}\right) \tau\right] \cos \left(\pi z+C_{2}\right) \\
& +O\left(\epsilon^{2}\right),
\end{aligned}
$$

where $\mathrm{Bo}_{H}=f \rho g_{y} H^{2} / \sigma$, with liquid density $\rho$. The form of Eq. (9) is recovered for $\mathrm{Bo}_{H}=0$ and it is observed that the column is unstable for $\mathrm{Bo}_{H}>1$. The order of magnitude predictions of settling times for slightly disturbed systems are possible by the suitable selection of $H$ and $L$. For example, $H$ could easily be taken as the vane height (in a propellant management device) and $L$ taken as the vane length. $C_{1}$ may be viewed as a weighting factor for the amplitude of the initial perturbation. 


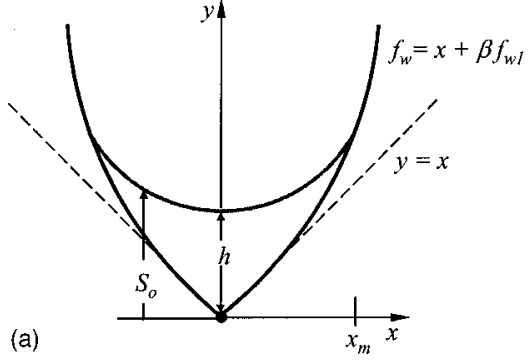

\section{INFINITE COLUMN SOLUTION: NONPLANAR WALLS}

The solution approach outlined above may be followed similarly for interior corners with slightly nonplanar walls. In the example provided here, the planar walls are symmetrically perturbed such that the corner half-angle condition at the corner vertex is preserved. The dimensional form of the wall perturbation is

$$
f_{w}^{\prime}=x^{\prime} \cot \alpha \pm B f_{w 1}^{\prime} .
$$

Nondimensionalizing using Table I quantities and choosing suitable scales for $B$ and $f_{w 1}^{\prime}$, Eq. (12) becomes

$$
f_{w}=x \pm \beta f_{w 1}+O\left(\beta^{2}\right),
$$

where $\beta \ll 1$. To maintain the corner half-angle condition at the vertex the form of $f_{w 1}$ must satisfy $\partial f_{w 1} /\left.\partial x\right|_{x=0}=0$. Examples of the nondimensional cross-flow sections for such non-planar walls are depicted in Fig. 5. A "concave wall" described by positive wall curvature is illustrated in Fig. 5(a), where $f_{w}=x+\beta f_{w 1}$. A "convex wall" described by negative wall curvature is depicted in Fig. 5(b), where $f_{w}$ $=x-\beta f_{w 1}$. In general, the Concus-Finn condition for these corners may depend on meniscus centerline height $h$ in addition to $\alpha$ and $\theta$. Depending on the choice of $f_{w 1}$, this condition may be computed using $\mathbf{n} \cdot \mathbf{k}=\cos \theta$, where $\mathbf{n}$ is the outward unit normal to $S_{0}$ and $\mathbf{k}$ is the inward unit normal to the corner walls. However, for both brevity and clarity, a perfectly wetting liquid $(\theta=0)$ will be assumed. This condition arises in many applications and assures corner wetting for all corner angles and wall curvatures independent of $h$. It also greatly simplifies all analytic expressions.

The solution procedure begins by expanding $R$ and $x_{m}$ in a like manner as $f_{w}$,

$$
\begin{aligned}
& R=R_{0}+\beta R_{1}+O\left(\beta^{2}\right), \\
& x_{m}=x_{m 0}+\beta x_{m 1}+O\left(\beta^{2}\right) .
\end{aligned}
$$

Equation (5) for $S_{0}$ is correct to $O(\epsilon)$ and may be used in conjunction with Eq. (13) to solve for the zeroth- and firstorder terms in Eqs. (14) and (15). Assuming a perfectly wetting fluid, the conditions of matched slope and surface height at $x_{m}$ must be satisfied, namely,

$$
\begin{aligned}
& \frac{\partial S_{0}}{\partial x}=\frac{\partial f_{w}}{\partial x}, \\
& S_{0}=f_{w},
\end{aligned}
$$

at $x=x_{m}$. Substituting Eqs. (5), (14), and (15) into (16) and (17), it is possible to show that to leading order,

$$
\begin{aligned}
& R=\left.h \mp \beta f_{w 1}\right|_{x_{m 0} 0}, \\
& x_{m}=\left.x_{m 0}\left[1 \pm \beta\left(\sin ^{2} \alpha \frac{\partial f_{w 1}}{\partial x}-h^{-1} f_{w 1}\right)\right]\right|_{x_{m 0}}, \\
& x_{m 0}=h(1+\sin \alpha) .
\end{aligned}
$$

At this point it is helpful to select a form for $f_{w 1}$ before proceeding. Choosing $f_{w 1}=x^{n}$, where $n>1$, and substituting into Eq. (18) yields

$$
\begin{aligned}
x_{m}= & h(1+\sin \alpha)\left[1 \mp \beta h^{n-1}(1+\sin \alpha)^{n-1}(1+\sin \alpha\right. \\
& \left.\left.-n \sin ^{2} \alpha\right)\right] .
\end{aligned}
$$

This form for $f_{w 1}$ requires that $B=\beta / H^{n-1} \tan ^{n} \alpha$ in Eq. (12). A second-order polynomial wall is achieved with $n$ $=2$ resulting in a further simplification of Eq. (19). This simplified problem will be analyzed here, which yields the system to $O(\beta)$,

$$
\begin{aligned}
f_{w}= & x \pm \beta x^{2}, \\
R= & h \mp \beta h^{2}(1+\sin \alpha)^{2}, \\
x_{m}= & h(1+\sin \alpha) \mp \beta h^{2}(1+\sin \alpha)^{2}(1+\sin \alpha \\
& \left.-2 \sin ^{2} \alpha\right) .
\end{aligned}
$$

To rederive the governing Eq. (4) for this problem it is necessary to compute $\left\langle w_{0}\right\rangle$ and $A$ for the cross-flow problem using

$$
\begin{aligned}
& \left\langle w_{0}\right\rangle=\frac{2}{A} \int_{0}^{x_{m}} \int_{f_{w}}^{s_{0}} w_{0} d y d x, \\
& A=2 \int_{0}^{x_{m}}\left(S_{0}-f_{w}\right) d x,
\end{aligned}
$$

where $w_{0}$ is determined from the $z$-component momentum equation for $\epsilon^{2} \ll 1$,

$$
\frac{\partial P_{0}}{\partial z}=\frac{\partial^{2} w_{0}}{\partial y^{2}} \sin ^{2} \alpha+\frac{\partial^{2} w_{0}}{\partial x^{2}} \cos ^{2} \alpha,
$$

subject to no slip along the walls, no shear stress on the free surface, and the symmetry condition along $x=0$, respectively:

$$
\begin{aligned}
& w_{0}=0, \quad \text { on } \quad y=f_{w}, \\
& \frac{\partial w_{0}}{\partial y}-\frac{\partial S_{0}}{\partial x} \frac{\partial w_{0}}{\partial x} \cot ^{2} \alpha=0, \quad \text { on } \quad y=S_{0},
\end{aligned}
$$




$$
\frac{\partial w_{0}}{\partial x}=0, \quad \text { on } \quad x=0
$$

The velocity $w_{0} \equiv-F_{\nu} \partial h / \partial z$ may be solved numerically for all values of $\alpha, \theta$, and $h$. However, as was the case for planar corners, it is anticipated that with the current scaling of the problem, asymptotic solutions or $F_{\nu}$ for all $\alpha$ and $\theta$ will be narrowly restricted. For example, for $\alpha^{2} \ll 1$, Eqs. (23)-(26) may be solved to show

$$
w_{0}=\frac{1}{2} \frac{\partial P_{0}}{\partial z}\left[x^{2}-y^{2}(1 \mp 2 \beta y)\right]
$$

and for $P_{0}=-1 / R$, the substitution of Eq. (20) leads to

$$
\frac{\partial P_{0}}{\partial z}=\frac{1}{h^{2}} \frac{\partial h}{\partial z}+O\left(\beta^{2}\right) .
$$

Substituting Eqs. (27) and (28) into (21) and performing the necessary integrations, the solution for $\left\langle w_{0}\right\rangle$ is

$$
\begin{aligned}
\left\langle w_{0}\right\rangle= & -\left[\frac{1}{6}+\frac{\alpha}{3}\left(1-\frac{5 \pi}{16}\right) \mp \frac{13}{45} \beta h\right] \frac{\partial h}{\partial z} \\
& +O\left(\alpha^{2}, \alpha \beta, \beta^{2}, \epsilon^{2}\right),
\end{aligned}
$$

from which it is observed that to leading order,

$$
\left\langle w_{0}\right\rangle=-\left(F_{I} \mp \frac{13}{45} \beta h\right) \frac{\partial h}{\partial z},
$$

where $F_{I}=F_{i}=F_{\nu}$ for the planar corner with $\alpha^{2} \ll 1$; see Table I. The dimensionless cross-flow area $A$ under these constraints, evaluated using Eq. (22), is

$$
A=h^{2}\left(1 \mp \frac{2}{3} \beta h\right)+O\left(\alpha^{2}, \alpha \beta, \beta^{2}, \epsilon^{2}\right) .
$$

Substituting Eqs. (29) and (30) into the leading-order governing equation (3) yields

$$
\begin{aligned}
& \frac{\partial}{\partial t}\left[\left(1 \mp \frac{2}{3} \beta h\right) h^{2}\right] \\
& \quad=-\frac{\partial}{\partial z}\left[-\left(1 \mp \frac{2}{3} \beta h\right)\left(F_{I} \mp \frac{13}{45} \beta h\right) h^{2} \frac{\partial h}{\partial z}\right] .
\end{aligned}
$$

Inspection of Eq. (31) shows that $F_{a}$ and $F_{\nu}$ are functions of $h$ for corners that are nonplanar. What is fortunate about Eq. (31), however, is that for the perturbed infinite column problem where $h=h_{0}+\epsilon h_{1}+O\left(\epsilon^{2}\right)$, with $h_{0}=1$, Eq. (31) simplifies to

$$
\frac{\partial h^{2}}{\partial t}=\left(F_{I} \mp \frac{13}{45} \beta\right) \frac{\partial}{\partial z}\left(h^{2} \frac{\partial h}{\partial z}\right)+O\left(\epsilon \beta, \alpha \beta, \alpha^{2}, \beta^{2}, \epsilon^{2}\right) .
$$

The prefactor on the right-hand side of Eq. (32) is $F_{\nu}$ for the nonplanar corners described. Rescaling time via $\tau=F_{\nu} t / 2$, Eq. (32) recovers the form of Eq. (4). Thus, the governing equation for the sinusoidally perturbed infinite fluid column is identical for both planar and slightly nonplanar solutions, with the difference coming only by way of modifications to the mean flow velocity parameter $F_{\nu}$, which appears as a scaling parameter. The $F_{\nu}$ values are listed in Table I for both problems and also apply in the case of gravity acting in the $y$ direction, Eq. (11). The net effect of nonplanar corners is to alter the geometric dependence of the rate of decay and to change the volumetric flow rate during settling. These quantities are provided below further discussion:

$$
\begin{aligned}
& \pi^{2} \tau=\epsilon \frac{\pi^{2}}{2} \frac{F_{\nu} \sin ^{2} \alpha}{f} \frac{\sigma t^{\prime}}{\mu L}=\epsilon \frac{\pi^{2}}{2}\left(F_{I} \mp \frac{13 \beta}{45}\right) \frac{\sin ^{2} \alpha}{f} \frac{\sigma t^{\prime}}{\mu L}, \\
& \dot{Q}^{\prime} \approx \pi \epsilon^{2} \frac{\sigma H^{2}}{\mu} \frac{\sin ^{3} \alpha}{f}\left(1 \mp \frac{2}{3} \beta \mp \frac{26}{15} \beta\right) \exp \left[-\pi^{2} \tau\right] .
\end{aligned}
$$

The inspection of Eq. (33) reveals that the time rate of decay decreases with increasing positive wall curvature, i.e., "concave wall" $f_{w}=x+\beta x^{2}$, Fig. 5(a). Conversely, the rate of decay increases with increasing negative wall curvature, i.e., "convex wall" $f_{w}=x-\beta x^{2}$, Fig. 5(b). This is attributable to a stronger dependence of the free surface curvature (driving force) on $h$.

An observation of Eq. (34) reveals that the amount of liquid participating during settling also increases for $h$ fixed with increasing negative wall curvature, $f_{w}=x-\beta x^{2}$, Fig. 5 (b). Not only is the driving force for flow increased, but the cross-flow area is also increased. The contribution of crossflow area and driving force of the mass flow rate are identified by the terms $2 \beta / 3$ and $26 \beta / 15$ in Eq. (34), respectively. Thus, the effect of changing driving force due to changing wall curvature may be shown to be 2.6 times larger than the effect of the changing cross-flow area for small corner angles, $\alpha^{2} \ll 1$. These general characteristics may be exploited to optimize corner geometries for specific applications.

\section{CONCLUDING REMARKS}

The asymptotic solutions presented above for a perturbed slender liquid column in an interior corner of infinite extent reveal the transient nature of the surface as it returns to its unperturbed state, driven by local gradients in surface curvature. The effect of gravity acting perpendicular to the corner axis is seen to accelerate, decelerate, or destabilize this process. A steady base state corner flow is shown to not affect the transient settling of small perturbations. Corner nonplanarity is shown to increase [Fig. 5(b)] or decrease [Fig. 5(a)] the rate of damped surface settling, depending on the sign of the wall curvature. For fixed mean liquid height $H$, the negative wall curvature [Fig. 5(b)] increases the volumetric flow rate, primarily through increases in the capillary driving force, but also through increases in cross-flow area. Transients computed analytically are minimized for a corner half-angle $\alpha=30$ and wetting angle $\theta=0$.

The predicted time constants presented are especially useful for a specific low-g fluid systems design. For example, for the sinusoidal disturbance depicted in Fig. 1, with $C_{1}$ $=1$ and $C_{2}=0$, Eq. (9) in dimensional terms reduces to 


$$
h^{\prime}=H\left[1+\epsilon \exp \left[-\pi^{2} \tau\right] \cos \left(\frac{\pi z^{\prime}}{L}\right)\right] .
$$

Thus, $98 \%$ of the disturbance is dissipated by $\tau=4 / \pi^{2}$, or in dimensional terms,

$$
t_{98 \%}^{\prime}=\frac{8}{\pi^{2}} \frac{L^{2}}{H} \frac{\mu}{\sigma} \frac{f}{F_{\nu} \sin ^{2} \alpha} .
$$

This is a convenient form for rapid calculation, noting $F_{\nu}$ $=\left[F_{i} \mp f(\beta)\right] . \quad f(\beta)=0$ for the planar corner; Fig. 2. For nonplanar corners, $f(\beta) \neq 0$; Fig. 5. For second-order polynomial corners with $\alpha^{2} \ll 1$ and perfectly wetting liquids, $f(\beta)=13 \beta / 45$. The impact of gravity modifies Eq. (35) such that

$$
t_{98 \%}^{\prime}=\frac{8}{\pi^{2}} \frac{L^{2}}{H} \frac{\mu}{\sigma} \frac{f}{F_{\nu} \sin ^{2} \alpha} \frac{1}{\left(1-\mathrm{Bo}_{H}\right)} .
$$

\section{ACKNOWLEDGMENTS}

This work was supported by NASA's Microgravity Science and Application Division through NASA Contract No.
NAS3-00126; project monitor, E. Ramé, NCMR, NASA Glenn Research Center. The author also wishes to thank S. Lichter.

${ }^{1} \mathrm{P}$. Concus and R. Finn, "On the behavior of a capillary free surface in a wedge," Proc. Natl. Acad. Sci. U.S.A. 63, 292 (1969).

${ }^{2}$ D. Langbein, "The shape and stability of liquid menisci at solid edges," J. Fluid Mech. 213, 251 (1990).

${ }^{3}$ M. M. Weislogel and S. Lichter, "Capillary flow in interior corners," J. Fluid Mech. 373, 349 (1998).

${ }^{4}$ T. C. Ransohoff, P. A. Gauglitz, and C. J. Radke, "Snap-off of gas bubbles in smoothly constricted noncircular capillaries," AIChE J. 33, 753 (1987).

${ }^{5} \mathrm{M}$. Dong and I. Chatzis, "The imbibition and flow of a wetting liquid along the corners of a square capillary tube," J. Colloid Interface Sci. 172, 278 (1995).

${ }^{6}$ D. Langbein and M. M. Weislogel, "Dynamics of liquids in edges and corners (DYLCO): IML-2 experiment for the BDPU," NASA TM 1998207916, 1998.

${ }^{7}$ P. S. Ayyaswamy, L. Catton, and D. K. Edwards, "Capillary flow in triangular grooves," ASME J. Appl. Mech. 41, 332 (1974).

${ }^{8}$ T. C. Ransohoff and C. J. Radke, "Laminar flow of a wetting liquid along corners of a predominantly gas-occupied noncircular pore," J. Colloid Interface Sci. 121, 392 (1988).

${ }^{9}$ M. M. Weislogel, "Capillary flow in an interior corner," NASA TM107364, Lewis Research Center, Cleveland, OH, 1996.

${ }^{10}$ S. F. Kistler, "Hydrodynamics of wetting," in Wettability, Surfactant Science Series Vol. 49, edited by J. C. Berg (Marcel Dekker, New York, 1993), p. 311. 
Physics of Fluids is copyrighted by the American Institute of Physics (AIP). Redistribution of journal material is subject to the AIP online journal license and/or AIP copyright. For more information, see http://ojps.aip.org/phf/phfcr.jsp 